International Journal of Modern Physics A

(C) World Scientific Publishing Company

\title{
A REVIEW OF RARE PION AND MUON DECAYS
}

\author{
DINKO POČANIĆ \\ Physics Department, University of Virginia, Charlottesville, VA 22904-4714, USA
}

Received (14 July 2004)

\begin{abstract}
After a decade of no measurements of pion and muon rare decays, PIBETA, a new experimental program is producing its first results. We report on a new experimental study of the pion beta decay, $\pi^{+} \rightarrow \pi^{0} e^{+} \nu$, the $\pi_{e 2 \gamma}$ radiative decay, $\pi^{+} \rightarrow e^{+} \nu \gamma$, and muon radiative decay, $\mu \rightarrow e \nu \bar{\nu} \gamma$. The new results represent four- to six-fold improvements in precision over the previous measurements. Excellent agreement with Standard Model predictions is observed in all channels except for one kinematic region of the $\pi_{e 2 \gamma}$ radiative decay involving energetic photons and lower-energy positrons.
\end{abstract}

Keywords: pi mesons; muons; rare decays.

\section{Motivation}

The PIBETA experiment 1 at the Paul Scherrer Institute (PSI) is a comprehensive set of precision measurements of the rare decays of the pion and the muon. The goals of the experiment's first phase are:

(i) To improve the experimental precision of the pion beta decay branching ratio, $\pi^{+} \rightarrow \pi^{0} e^{+} \nu$ (also referred to as $\pi_{e 3}$, or $\pi_{\beta}$ ), from the present $\sim 4 \%$ to $\sim 0.5 \%$.

(ii) To measure the branching ratio of the radiative pion decay $\pi \rightarrow e \nu \gamma\left(\pi_{e 2 \gamma}\right.$, or $\mathrm{RPD}$ ), enabling a precise evaluation of the pion axial-vector form factor $F_{A}$, and limits on the tensor form factor $F_{T}$, predicted to vanish in the Standard Model (SM).

(iii) An extensive measurement of the radiative muon decay rate, $\mu \rightarrow e \nu \bar{\nu} \gamma$, with broad phase space coverage, enabling a search for non- $(\mathrm{V}-\mathrm{A})$ admixtures in the weak Lagrangian.

The experiment's second phase calls for a precise measurement of the $\pi \rightarrow e \nu$ (known as $\pi_{e 2}$ ) decay rate, used for normalization in the first phase. The current $0.33 \%$ accuracy would be improved to under $0.2 \%$, in order to provide a precise test of lepton universality, and, hence, of certain extensions to the Standard Model. In this report we focus mainly on parts (i) and (ii) above, and briefly discuss the first muon radiative results, (iii).

The rare pion beta decay, $\pi^{+} \rightarrow \pi^{0} e^{+} \nu$ (branching ratio $R_{\pi \beta} \simeq 1 \times 10^{-8}$ ), is one of the most basic semileptonic electroweak processes. It is a pure vector transition 
between two spin-zero members of an isospin triplet, and is therefore analogous to superallowed Fermi (SF) transitions in nuclear beta decay. The conserved vector current (CVC) hypothesis 23 and quark-lepton universality relate the rate of the pure vector pion beta decay to that of muon decay via the Cabibbo-Kobayashi-Maskawa (CKM) quark mixing matrix element $V_{u d} \underline{45}$ in a theoretically exceptionally clean way ${ }^{67}$ Hence, pion beta decay presents an excellent means for a precise experimental determination of the CKM matrix element $V_{u d}, \frac{789110}{18}$ hindered only by the low branching ratio of the decay.

The CKM quark mixing matrix has a special significance in modern physics as a cornerstone of a unified description of the weak interactions of mesons, baryons and nuclei. In a universe with three quark generations the $3 \times 3$ CKM matrix must be unitary, barring certain classes of hitherto undiscovered processes not contained in the Standard Model. Thus, an accurate experimental evaluation of the CKM matrix unitarity provides an independent check of possible deviations from the SM. As the best studied element of the CKM matrix, $V_{u d}$ plays an important role in all tests of its unitarity. However, evaluations of $V_{u d}$ from neutron decay have, for the most part, not been consistent with results from nuclear SF decays 11 Clearly, a precise evaluation of $V_{u d}$ from pion beta decay, the theoretically cleanest choice, is of interest.

Radiative pion decay offers unparalleled access to information on the pion's structure. Given the unique role of the pion as the quasi-Goldstone boson of the strong interaction, the implications are far reaching. In the Standard Model description 12 of the $\pi^{+} \rightarrow e^{+} \nu \gamma$ decay, where $\gamma$ is a real or virtual photon $\left(e^{+} e^{-}\right.$ pair), the decay amplitude $\mathcal{M}$ depends on the vector $\mathrm{V}$ and axial vector $\mathrm{A}$ weak hadronic currents. Both currents give rise to structure-dependent terms $\mathrm{SD}_{\mathrm{V}}$ and $\mathrm{SD}_{\mathrm{A}}$ associated with virtual hadronic states, while the axial-vector current alone causes the inner bremsstrahlung process IB from the pion and positron. The IB contribution to the decay probability can be calculated in a straightforward manner using QED methods. The structure-dependent amplitude is parameterized by the vector form factor $F_{V}$ [constrained by CVC to $0.0259(5)$ ] and the axial vector form factor $F_{A}$ that have to be extracted from experiments.

The ratio $\gamma=F_{A} / F_{V}$ in $\pi \rightarrow e \nu \gamma$ decay directly determines the chiral perturbation theory parameter sum $\left(l_{9}+l_{10}\right)$, or, equivalently, $\alpha_{E}$, the pion polarizability. 242526 These quantities are of longstanding interest since they are among the few unambiguous predictions of chiral symmetry and QCD at low energies. The current status of these measurements is not satisfactory, as there is considerable scatter among the various experimental determinations of $F_{A} / F_{V}$, and the accepted Particle Data Group (PDG) average value has a $14 \%$ uncertainty 11

Moreover, the statistical accuracy of the present experimental data on the radiative pion decay 131415161718 cannot rule out contributions from other allowed terms in the interaction lagrangian, namely the scalar S, pseudoscalar P, and tensor $\mathrm{T}$ admixtures 19 Nonzero values of any of these amplitudes would imply new physics outside the Standard Model. In particular, reports from the ISTRA 
collaboration 2021 have indicated a nonzero tensor term, $F_{T}=-0.0056(17)$. A careful analysis by Herczeg of the existing beta decay data set could not rule out such a value of $F_{T}$, which he presumed could be due to leptoquarks22. In contrast, Chizhov proposed a new intermediate chiral boson with an anomalous interaction with matter, in order to account for the apparent non- $(\mathrm{V}-\mathrm{A})$ behavior in RPD 23

Finally, radiative muon decay (RMD) provides an even better testing ground for non- $(\mathrm{V}-\mathrm{A})$ interaction terms than does RPD, thanks to the absence of internal structure in the muon. Measuring photon-positron energy distributions in RMD allows an evaluation of the $\mu^{+}$decay parameter $\bar{\eta}$ that is predicted to be zero in the V-A Standard Model. The current limit on $\bar{\eta}$ is loose, $0.02 \pm 0.08$. It is of considerable interest to bring the uncertainties down to a level competitive with the constraints from standard muon decay.

\section{Experimental Method}

The PIBETA apparatus is a large-acceptance non-magnetic detector optimized for detection of photons and electrons in the energy range of $5-150 \mathrm{MeV}$ with high efficiency, energy resolution and solid angle. The main sensitive components of the apparatus, shown and labeled in Fig. 1 are:

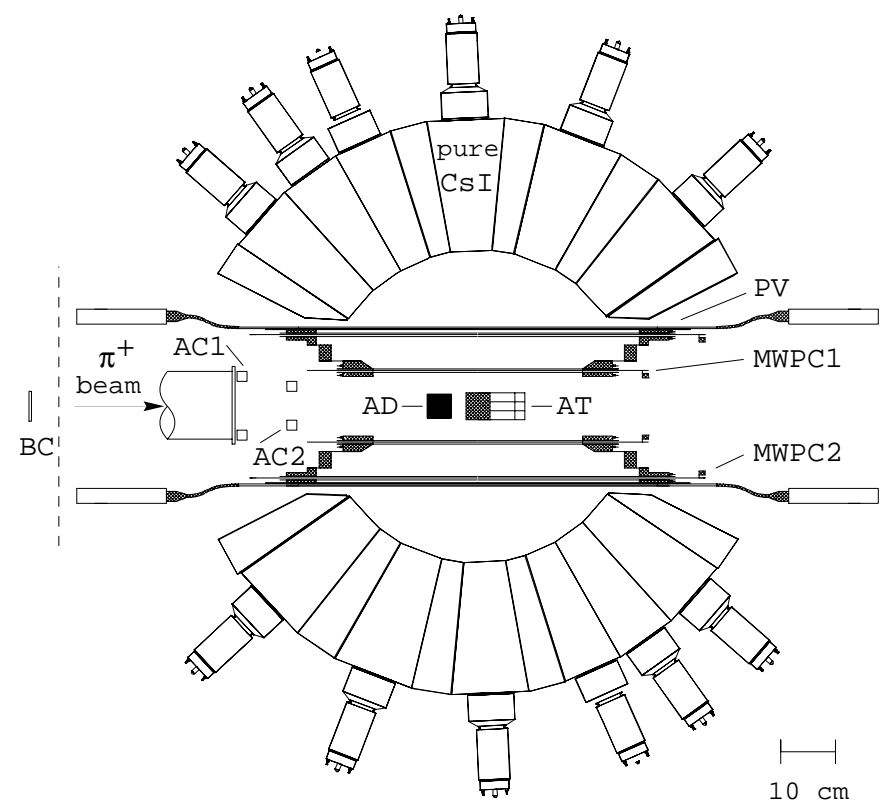

Fig. 1. Schematic cross section of the PIBETA apparatus showing the main components: beam entry counters (BC, AC1, AC2), active degrader (AD), active target (AT), wire chambers (MW$\mathrm{PCs}$ ) and support, plastic veto (PV) detectors and PMTs, pure CsI calorimeter and PMTs. 
(i) beam defining plastic scintillator detectors: $\mathrm{BC}$, a thin forward beam counter, $\mathrm{AC}_{1}$ and $\mathrm{AC}_{2}$, cylindrical active collimators, $\mathrm{AD}$, an active degrader, $\mathrm{AT}$, a 9-element segmented active target that stops the $\pi^{+}$beam;

(ii) charged particle tracking and id: $\mathrm{MWPC}_{1}$ and $\mathrm{MWPC}_{2}$, cylindrical chambers, and PV, a 20-bar segmented thin plastic scintillator hodoscope;

(iii) a 240-element segmented spherical pure-CsI shower calorimeter, subtending a solid angle of $\sim 80 \%$ of $4 \pi$.

The entire system is enclosed in a temperature-controlled $\mathrm{Pb}$ house lined with cosmic muon veto detectors.

To collect the available $\pi_{b}$ eta decay events we recorded all non-prompt largeenergy (above the $\mu \rightarrow e \nu \bar{\nu}$ endpoint) electromagnetic shower pairs occurring in opposite detector hemispheres (non-prompt two-arm events). In addition, we recorded a large prescaled sample of non-prompt single shower (one-arm) events. Using these minimum-bias sets, we extract $\pi_{\beta}$ and $\pi_{e 2}$ events, using the latter for normalization. In a stopped pion experiment these two channels have nearly the same detector acceptance, and have much of the systematics in common.

These two event classes are part a full complement of twelve fast analog triggers comprising all relevant logic combinations of one- or two-arm, low- or high calorimeter threshold, prompt and delayed (with respect to $\pi^{+}$stop time), as well as a random and a three-arm trigger, all of which were implemented in order to obtain maximally comprehensive and unbiased data samples. Details of the method are explained in detail in Ref. 27. The list of decays measured and/or used for normalization in our work is given in Table 1

Table 1. List of decays measured in the PIBETA experiment, along with the corresponding branching ratios.

\begin{tabular}{llcl}
\hline \multicolumn{1}{c}{ Decay } & Branching ratio & \\
\hline$\pi^{+} \rightarrow \quad \mu^{+} \nu$ & 1.0 & \\
& $\mu^{+} \nu \gamma$ & $\sim 2.0 \times 10^{-4}$ & \\
& $\mathrm{e}^{+} \nu$ & $\sim 1.2 \times 10^{-4}$ & normalize to \\
& $\mathrm{e}^{+} \nu \gamma$ & $\sim 5.6 \times 10^{-8}$ & measure \\
& $\pi^{0} \mathrm{e}^{+} \nu$ & $\sim 1.0 \times 10^{-8}$ & measure \\
$\pi^{0} \rightarrow \quad \gamma \gamma$ & $\sim 0.9880$ & measure \\
& $\mathrm{e}^{+} \mathrm{e}^{-} \gamma$ & $\sim 1.2 \times 10^{-2}$ & measure \\
& $\mathrm{e}^{+} \mathrm{e}^{-} \mathrm{e}^{+} \mathrm{e}^{-}$ & $\sim 3.1 \times 10^{-5}$ & \\
$\mathrm{e}^{+} \mathrm{e}^{-}$ & $\sim 6.2 \times 10^{-8}$ & \\
$\mu^{+} \rightarrow \quad$ & $\mathrm{e}^{+} \nu \bar{\nu}$ & 1.0 & normalize to \\
& $\mathrm{e}^{+} \nu \bar{\nu} \gamma$ & $\sim 0.014$ & measure \\
& $\mathrm{e}^{+} \nu \bar{\nu} \mathrm{e}^{+} \mathrm{e}^{-}$ & $\sim 3 \times 10^{-5}$ & measure \\
\hline
\end{tabular}




\section{Results}

The building and testing of the detector components were completed in 1998, followed by the assembly and commissioning of the full detector apparatus. Data acquisition with the PIBETA detector started in the second half of 1999, initially at a reduced pion stopping rate, as planned. The experiment ran subsequently during 2000 and 2001 at $\sim 1 \mathrm{MHz} \pi^{+}$stopping rate. We review below the current status of the analysis of the data acquired during this running period.

\subsection{Pion beta decay, $\pi^{+} \rightarrow \pi^{0} e^{+} \nu$}

In spite of its low branching ratio, the pion beta decay signal, marked by a nearly back-to-back energetic pair of neutral showers from the $\pi^{0} \rightarrow \gamma \gamma$ decay, is strong and without appreciable background. This is demonstrated in Fig. 2 which shows virtually background-free histograms of $\gamma-\gamma$ relative timing, and of the event time distribution following the pion stop time, $t$ (DEG). The corresponding CsI calorimeter energy distributions for the $\pi_{\beta}$ events and for the normalizing $\pi_{e 2}$ events are also given in the same figure We note the excellent agreement between the measured distributions and those simulated by the Monte Carlo program GEANT3.
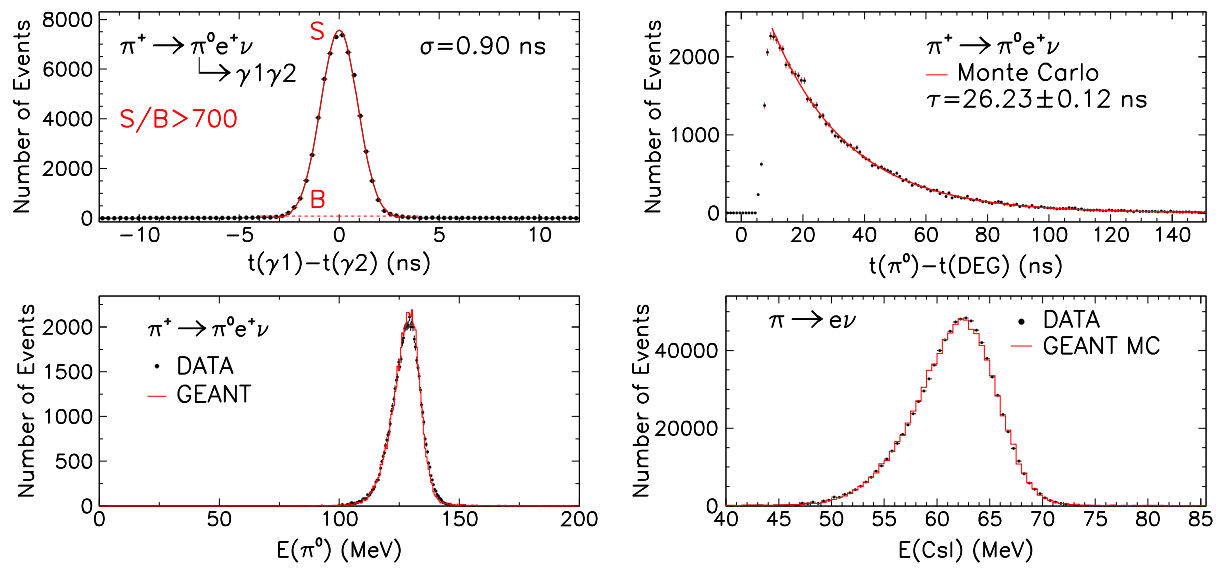

Fig. 2. Histogram of $\gamma-\gamma$ time differences for $\pi_{\beta}$ decay events (dots); curve: fit with a Gaussian function plus a constant (top left). Histogram of time differences between the beam pion stop and the $\pi_{\beta}$ decay events (dots); curve: pion lifetime (top right). CsI calorimeter energy spectra for the pion beta decay events (bottom left) and the normalizing decay $\pi_{2 e}$ (bottom right).

Details of the analysis method of the $\pi_{\beta}$ decay channel can be found in Refs. 28 and 29. Applying our method and using the PDG 2004 recommended value of $R_{\pi e 2}^{\exp }=1.230(4) \times 10^{-4}, 11$ we extract the pion beta decay branching ratio:

$$
R_{\pi \beta}^{\exp }=\left[1.036 \pm 0.004 \text { (stat) } \pm 0.004 \text { (syst) } \pm 0.003\left(\pi_{\mathrm{e} 2}\right)\right] \times 10^{-8},
$$


or, in terms of the decay rate,

$$
\Gamma_{\pi \beta}^{\exp }=\left[0.3980 \pm 0.0015(\text { stat }) \pm 0.0015(\text { syst }) \pm 0.0013\left(\pi_{\mathrm{e} 2}\right)\right] \mathrm{s}^{-1} .
$$

In both expressions the first uncertainty is statistical, the second systematic, and the third is the $\pi_{e 2}$ branching ratio uncertainty. Our result represents a six-fold improvement in accuracy over the most precise previous measurement 30 . Furthermore, our result is in excellent agreement with predictions of the SM and CVC given the PDG recommended value range for $V_{u d}$ [11

$$
R_{\pi \beta}^{\mathrm{SM}}=(1.038-1.041) \times 10^{-8} \quad(90 \% \text { C.L. }),
$$

and represents the most accurate test of CVC and Cabibbo universality in a meson to date. Our result confirms the validity of the radiative corrections for the process at the level of $4 \sigma_{\exp }$, since, excluding loop corrections, the SM would predict $R_{\pi \beta}^{\text {no rad. corr. }}=(1.005-1.007) \times 10^{-8}$ at $90 \%$ C.L.

Using our measured branching ratio $R_{\pi \beta}^{\exp }$, we can calculate a new value of $V_{u d}$ from pion beta decay, $V_{u d}^{(\mathrm{PIBETA})}=0.9728(30)$, which is in excellent agreement with the PDG 2004 average, $V_{u d}^{\left(\mathrm{PDG}^{\prime} 04\right)}=0.9738(5)$. We will continue to improve the overall accuracy of the $\pi \beta$ decay branching ratio to $\sim 0.5 \%$ by further refining the experiment simulation and analysis, and by adding new data.

\subsection{Radiative pion decay, $\pi^{+} \rightarrow e^{+} \nu \gamma$}

During our 1999-2001 run we recorded over 40,000 $\pi_{e 2 \gamma}$ radiative pion decays (RPD) events. Besides its intrinsic interest, the $\pi_{e 2 \gamma}$ process is an important physics background to other decays under study, particularly the $\pi_{\beta}$ decay. The pion radiative decay analysis has given us the most surprising result to date, and has commanded significant effort on our part to resolve the issue.

The different event triggers used in our experiment are sensitive to three distinct regions in the $\pi_{e 2 \gamma}$ phase space:

- region $\mathrm{A}$ with $e^{+}$and $\gamma$ emitted into opposite hemispheres, each with energy exceeding that of the Michel edge $\left(E_{M} \simeq 52 \mathrm{MeV}\right)$, recorded in the main two-arm trigger,

- region B with an energetic photon $\left(E_{\gamma}>E_{M}\right)$, and $E_{e+} \geqslant 20 \mathrm{MeV}$, recorded in the one-arm trigger, and

- region $\mathrm{C}$ with an energetic positron $\left(E_{e+}>E_{M}\right)$, and $E_{\gamma} \geqslant 20 \mathrm{MeV}$, also recorded in the one-arm trigger.

Together, the three regions overconstrain the Standard Model parameters describing the decay, and thus allow us to examine possible new information about the pion's hadronic structure, or non- $(\mathrm{V}-\mathrm{A})$ interactions. The RPD data are of a similar quality to our $\pi_{\beta}$ event set, particularly in region $\mathrm{A}$, as is readily verified in Fig. 3. which shows histograms of $e-\gamma$ time differences and event timing with 

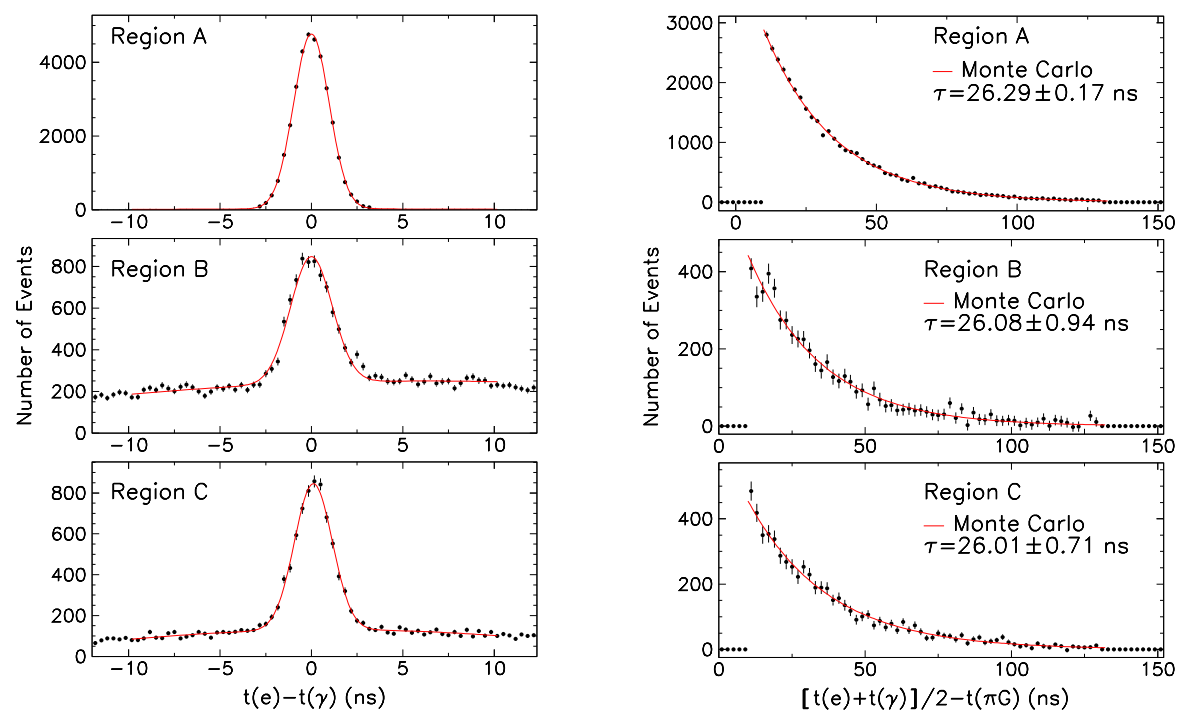

Fig. 3. $e-\gamma$ timing difference for $\pi \rightarrow e \nu \gamma$ decay events in regions A, B, and C (left panels, top to bottom, respectively). The right panels plot the $\pi^{+} \rightarrow e^{+} \nu \gamma$ event timing relative to the $\pi^{+}$ stop gate time, $t(\pi \mathrm{G})$, after accidental background subtraction. Monte Carlo decay functions are shown as full lines; best-fit values for the pion lifetime are indicated for each region.

respect to the pion stop gate time, $t(\pi \mathrm{G})$. The analysis of these data is involved; more details can be found in Refs. 31 and 1 .

The dependence of the region- $A$ experimental and theoretical branching ratio on the value of $\gamma$ is shown in Fig. 4 (left), indicating two solutions. The positive $\gamma$ solution is preferred by a $\chi^{2}$ ratio of $\sim 50: 1$ once data from regions $B$ and $C$ are included in the analysis (right panel). We compare the experimental and theoretical branching ratios for the three phase space regions in Table 2 We note that due to the large statistical and systematic uncertainties present in all older experiments, our values are consistent with previously published measurements. The best CVC fit to our data yields

$$
\gamma=0.443 \pm 0.015, \quad \text { or } \quad F_{A}=0.0115(4) \quad \text { with } \quad F_{V} \equiv 0.0259 .
$$

This result represents a four-fold improvement in precision over the previous world average $F_{A}=0.0116(16)$ 11. It is consistent with chiral Lagrangian calculations 322526 , and will lead to a correspondingly improved precision in the order $p^{4}$ chiral constant $l_{10} 3326$.

Thus, our experimental $\pi^{+} \rightarrow e^{+} \nu \gamma$ branching ratios and energy distributions in kinematic regions $A$ and $C$ are compatible with the $(V-A)$ interaction. The sizable $19 \%$ shortfall in the measured branching ratio in region $B$ dominates the high value of $\chi^{2} /$ d.o.f $=25.4$ (Table 21), and is disconcerting. In a fit restricted to region $A$ data only, we obtain $\gamma=0.480 \pm 0.016$; this result remains unchanged if region $C$ data are added to the fit. Significantly, all previous studies except one ${ }^{18}$ (which, 

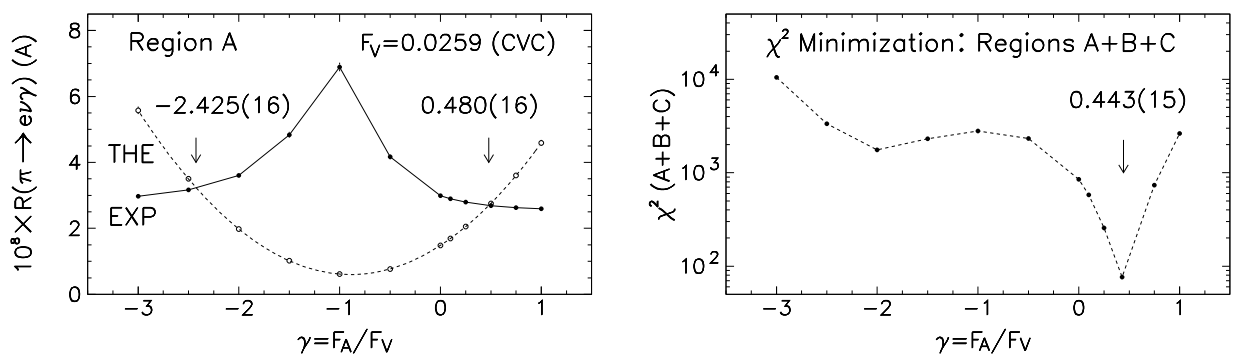

Fig. 4. Left plot: $\pi^{+} \rightarrow e^{+} \nu \gamma$ branching ratio values as a function of $\gamma \equiv F_{A} / F_{V}$. The theoretical parabola follows from the $V-A$ model). The experimental values reflect fits to region $A$ data only. Right plot: minimum $\chi^{2}$ values of simultaneous fits to the entire data set (regions $A, B, C$ ).

Table 2. Best-fit $\pi \rightarrow e \nu \gamma$ branching ratios.

\begin{tabular}{ccccc}
\hline $\begin{array}{c}E_{e^{+}}^{\min } \\
(\mathrm{MeV})\end{array}$ & $\begin{array}{c}E_{\gamma}^{\min } \\
(\mathrm{MeV})\end{array}$ & $\theta_{e \gamma}^{\min }$ & $\begin{array}{c}R_{\exp } \\
\left(\times 10^{-8}\right)\end{array}$ & $\begin{array}{c}R_{\text {the }} \\
\left(\times 10^{-8}\right)\end{array}$ \\
\hline 50 & 50 & - & $2.71(5)$ & $2.583(1)$ \\
10 & 50 & $40^{\circ}$ & $11.6(3)$ & $14.34(1)$ \\
50 & 10 & $40^{\circ}$ & $39.1(13)$ & $37.83(1)$ \\
\hline
\end{tabular}

too, found an anomaly), have analyzed only data with kinematics compatible with our region $A$. An illustration of the nature of the observed discrepancy is given in the region- $B$ plots of a conveniently defined kinematic variable $\lambda$ in Fig. 5 . Addition of a small negative tensor term, $F_{T} \sim-0.002$, improves agreement with the data.

As of this writing the PIBETA collaboration is pursuing a dedicated run to determine the extent and nature of the observed discrepancy more precisely.
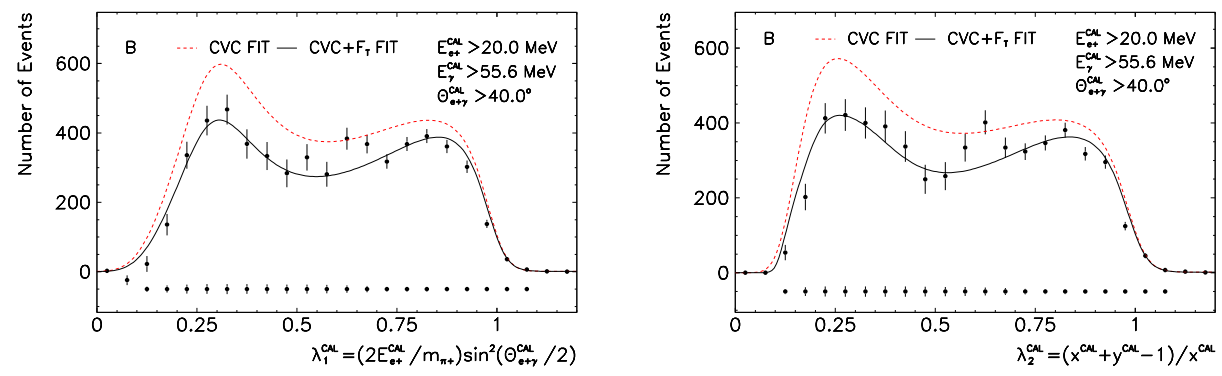

Fig. 5. Left panel: measured spectrum of $\lambda_{1}=\left(2 E_{e} / m_{\pi}\right) \sin ^{2}\left(\theta_{e \gamma} / 2\right)$ in $\pi_{e 2 \gamma}$ decay for the kinematic region $B$, with limits noted in the figure. Dashed curve: three-region global best fit with the pion form factor $F_{V}=0.0259$ fixed by the CVC hypothesis, $F_{T}=0$, and $F_{A}$ free. Solid curve: $F_{V}=0.0259$ and $F_{A}=0.0115$ from the first fit, this time with $F_{T}$ released to vary freely, resulting in $F_{T}=-0.0018(3)$. Error bars on the points at bottom of graph reflect the expected uncertainties in the proposed dedicated measurement. Bottom panel: same as above, but plotting the variable $\lambda$ evaluated purely on the basis of photon and positron energies: $\lambda_{2}=(x+y-1) / x$, where $x=2 E_{\gamma} / m_{\pi}$, and $y=2 E_{e} / m_{\pi}$. The two methods agree well. 


\subsection{Radiative muon decay, $\mu^{+} \rightarrow e^{+} \nu \bar{\nu} \gamma$}

The 1999-2001 run produced a set of some 300,000 radiative muon decay events, increasing the world set by two orders of magnitude. Due to an emphasis in the early analysis on the pion rare decay channels, the RMD analysis has not yet reached the same sub- $1 \%$ level of precision. The analysis is currently at the $\sim 1 \%$ level and both integrated and differential branching ratios are in good agreement with the $\mathrm{V}-\mathrm{A}$

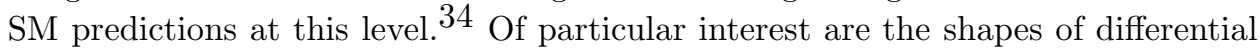
distributions of $\Delta$, a suitably defined kinematic variable (analogous to $\lambda$ in RPD), shown in Fig. 6 Clearly, nothing like the $\sim 20 \%$ discrepancy of region $B$ in RPD is observed here.
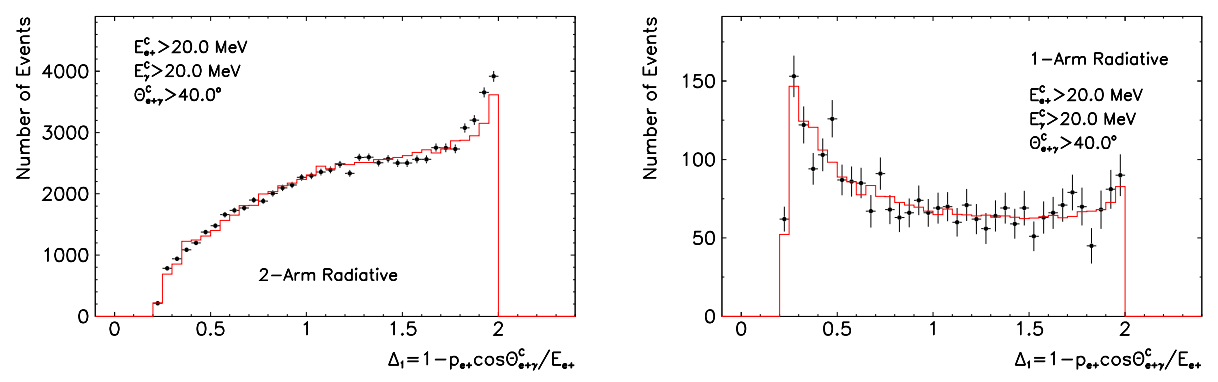

Fig. 6. Dots: distributions of the kinematic variable $\Delta=1-p_{e} \cos \theta_{e \gamma} / E_{e}$ for radiative muon decay events collected with the two-arm (left) and one-arm trigger (right). Histograms: Standard Model V-A GEANT simulation.

This work is in progress, and the 2004 dedicated RPD run is expected to more than double the RMD event set, adding high-quality new data. Interpretation of the PIBETA RMD data below the $1 \%$ accuracy level will not be possible without new reliable radiative corrections for this process.

\section{Summary}

Over a decade after the last precision measurements of the rare pion and muon decays were completed, the PIBETA experiment at PSI is revisiting the field, and producing new results. Unlike any experiment exploring the field before it, the PIBETA project measures simultaneously practically all of the rare $\pi$ and $\mu$ decays. This gives it an unusually powerful set of built-in consistency checks.

The pion beta decay precision has been improved six-fold, resulting in a first definitive test of the CVC and radiative corrections in a meson. Work on this decay channel is continuing, and will reach $\sim 0.5 \%$ accuracy in this first phase of the project. The current result, with a combined systematic and statistical uncertainty of $0.64 \%$ is in excellent agreement with the SM predictions.

The PIBETA radiative pion decay measurements cover a broader region of phase space than previous experiments, with more than an order of magnitude higher 
statistics, and have brought about a four-fold improvement in the precision of the pion axial form facto. However, the unexpected and pronounced deviation from the $\mathrm{V}-\mathrm{A}$ description of the process is limiting this precision, and raising important questions. This matter is being addressed in a current dedicated run.

Comparable improvements in precision are expected in radiative muon decay.

Finally, in the following, second phase of the project, the PIBETA collaboration will turn its attention to the $\pi \rightarrow e \nu$ process which provides the best test of lepton universality and a selective check on possible new physics.

This material is based upon work supported by the National Science Foundation under Grants No. 0098758 and 0354808.

\section{References}

1. PIBETA home page: http://pibeta.phys.virginia.edu/

2. S. S. Gershtein and I. B. Zel'dovich, Zh. Eksp. Teor. Fiz. 29, 698.

3. R. P. Feynman and M. Gell-Mann, Phys. Rev. 109, 193 (1958).

4. N. Cabibbo, Phys. Rev. Lett. 10, 531 (1963).

5. M. Kobayashi and T. Maskawa, Prog. Theor. Phys. 49, 652 (1973).

6. G. Källèn, Elementary Particle Physics, (Addison-Wesley, Reading, Mass.,1964).

7. A. Sirlin, Rev. Mod. Phys. 50, 573 (1978), [erratum: ibid., 50, 905 (1978)].

8. A. Sirlin, Nucl. Phys. B 196, 83 (1982).

9. W. Jaus, Phys. Rev. D 63, 053009 (2001).

10. V. Cirigliano, M. Knecht, H. Neufeld, and H. Pichl, Eur. Phys. J. C 27, 255 (2003).

11. S. Eidelman et al., Phys. Lett. B 592, 1 (2004).

12. D. A. Bryman, P. Depommier, and C. Leroy, Phys. Rep. 88 (1982) 151.

13. P. Depommier, et al., Phys. Lett. 7 (1963) 285.

14. A. Stetz, et al., Phys. Rev. Lett. 33 (1974) 1455.

15. A. Stetz, et al., Nucl. Phys. B138 (1978) 285.

16. A. Bay, et al., Phys. Lett. B174 (1986) 445.

17. L. E. Piilonen, et al. Phys. Rev. Lett. 57 (1986) 1402.

18. V. N. Bolotov, et al., Phys. Lett. B243 (1990) 308; Nucl. Phys. B16 (1990) 459.

19. K. Mursula and F. Schenk, Nucl. Phys. B253 (1985) 189.

20. A. A. Poblaguev, Phys. Lett. B238 (1990) 108; ibid., B286 (1992) 169.

21. A. A. Poblaguev, Phys. Rev. D 68, 054020 (2003).

22. P. Herczeg, Phys. Rev. D 49 (1994) 247.

23. M. V. Chizhov, Mod. Phys. Lett. A8 (1993) 2753, hep-ph/0307100 hep-ph/0310203

24. B.R. Holstein, Comments Nucl. Part. Phys. 19 (1990) 221.

25. J. Bijnens and P. Talavera, Nucl. Phys. B489 (1997) 387.

26. C. Q. Geng, I.-L. Ho, and T. H. Wu, Nucl. Phys. B 684, 281 (2004).

27. E. Frlež et al., Nucl. Inst. \& Meth. in Phys. Res. B489 (2004) 300.

28. D. Počanić et al., e-Print hep-ex/0312030

29. Weidong Li, Ph.D. thesis, University of Virginia, 2004.

30. W K McFarlane, et al., Phys. Rev. D 32, 547 (1985).

31. E. Frlež et al., e-Print hep-ex/0312029

32. B. R. Holstein, Phys. Rev. D 33, 3316 (1986).

33. G. Amoros et al., Nucl. Phys. B 602, 87 (2001).

34. E. Frlež et al., hep-ex/0312025 